\title{
Aminomethylation of Chlorophyll a Phorbine Derivatives Using $\operatorname{Bis}(N, N$-dimethylamino)methane
}

\author{
Dmitry V. Belykh, ${ }^{\mathrm{a} @}$ Irina S. Tarabukina, ${ }^{\mathrm{a}}$ Ivan V. Gruzdev, ${ }^{\mathrm{b}}$ \\ and Alexander V. Kuchin ${ }^{\mathrm{a}}$
}

${ }^{a}$ Institute of Chemistry, Komi Scientific Centre, Ural Division of Russian Academy of Sciences, 167982 Syktyvkar, Russia ${ }^{\mathrm{b}}$ Institute of Biology, Komi Scientific Centre, Ural Division of Russian Academy of Sciences, 167982 Syktyvkar, Russia

${ }^{\circledR}$ Corresponding authorE-mail: belykh-dv@chemi.komisc.ru,belykh-dv@mail.ru

\begin{abstract}
It has been shown that aminomethylation of the vinyl group in Zn complexes of chlorophyll a phorbine derivatives can be accomplished under the action of bis(N,N-dimethylamino)methane in the presence of weak acid (AcOH) at room temperature. The new chlorophyll a phorbine derivatives with two N,N-dimethylaminomethyl substituents in vinyl group were synthesized.
\end{abstract}

Keywords: Methylpyropheophorbide $a$, methylpyropheophorbide $a$ 13(1)-oxim, methylpyropheophorbide $a$ 13(1)-methoxim, zinc complexes, aminomethylation, bis( $N, N$-dimethylamino)methane.

\section{Introduction}

The insertion of dimethylaminomethyl groups into the molecule of natural chlorins is of interest for the synthesis of cationic photosensitizers (PS) for the photodynamic therapy (PDT) in oncology - the alkylation of these groups by methyl iodide represents a convenient route to cationic substituent. ${ }^{[1,2]}$ Earlier we have devised a

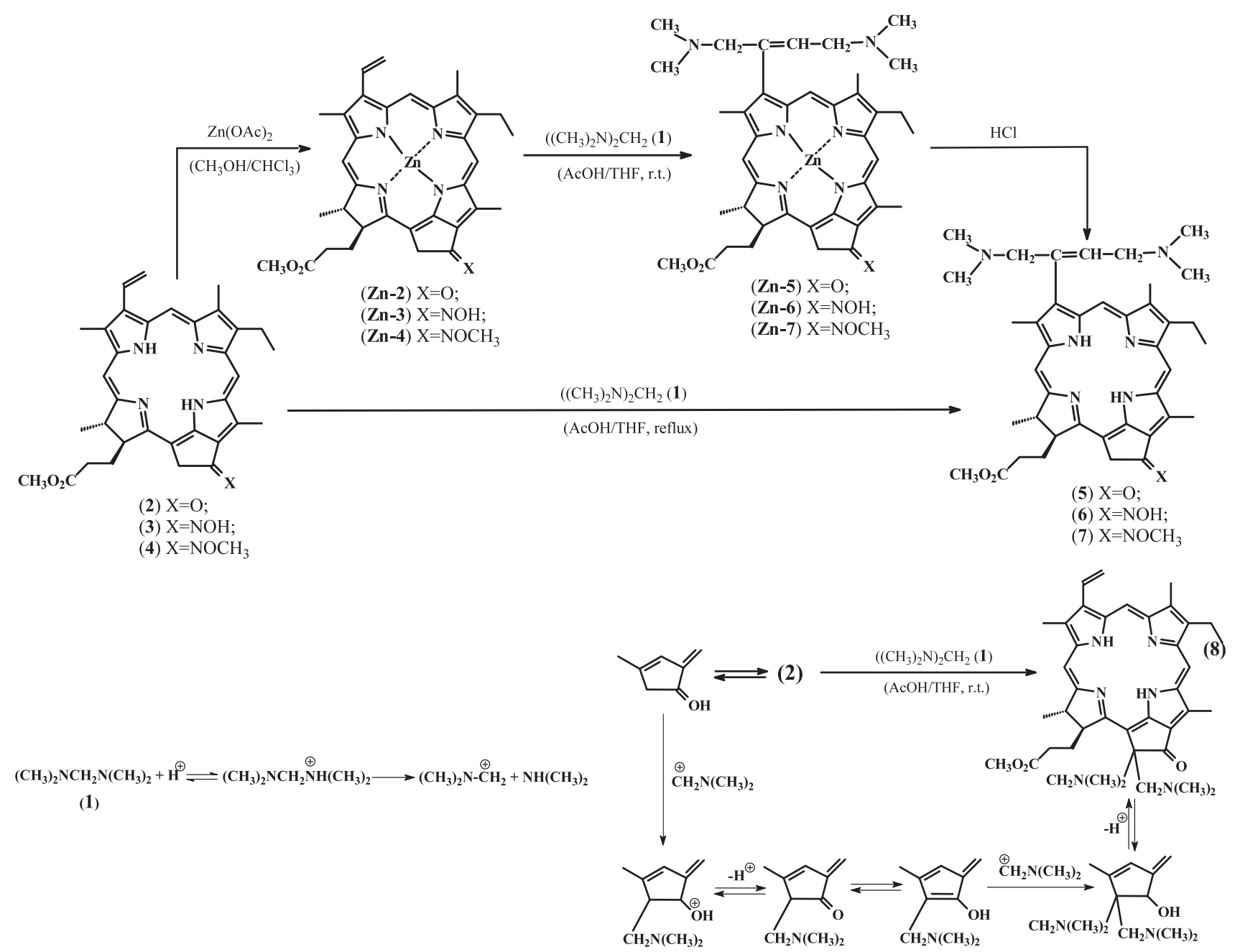

Scheme 1. 
method of aminomethylation of 13-amides $\mathrm{e}_{6},{ }^{[3]}$ allowing the insertion of two dimethylaminomethyl substituents into vinyl group. According to this procedure the initial vinyl-chlorin was refluxed with bis( $N, N$-dimethylamino) methane (1) (Scheme 1) in the mixture of tetrahydrofuran (THF) with acetic acid ( $\mathrm{AcOH})$ and the corresponding twice aminomethylated derivatives were obtained with $60-80 \%$ yields. To reveal the new opportunities of bis $(N, N-$ dimethylamino)methane (1) as an aminomethylating agent in the chemistry of natural chlorins, we have studied its interaction with some phorbine derivatives of chlorophyll $a$ (Scheme 1).

\section{Experimental}

${ }^{1} \mathrm{H}$ NMR spectra were recorded in $\mathrm{CDCl}_{3}$ on a Bruker Avance III spectrometer at $300 \mathrm{MHz}$. IR spectra were measured on a Shimadzu IR Prestige 21 instrument in $\mathrm{KBr}$ disks. EI mass spectra were obtained on DSQ spectrometer (Thermo, Direct Probe System), ionization voltage $70 \mathrm{eV}$. Electrospray mass spectra were obtained on Thermo LCQ Fleet spectrometer. UVvis spectra were recorded for solutions in $\mathrm{CHCl}_{3}$ on an UV-1700 spectrometer (PharmaSpec, Shimadzu) in the 200-1100 nm range, using $1 \mathrm{~cm}$ quartz cuvets. The reaction control was performed by TLC on Silufol plates, eluent $\mathrm{CCl}_{4}$ :acetone (1:4 by volume). Alfa Aesar $70 / 230 \mu$ silica was used for the column chromatography. Methylpyropheophorbide $a$ (2) was obtained as described elswere ${ }^{[4]}$ from methylpheophorbide $a$ which was prepared according to the known procedure. ${ }^{[5]}$

Methylpyropheophorbide a 13(1)-oxim, 3. A solution of methylpyropheophorbide $a$ (2) (144 mg, $0.26 \mathrm{mmol})$ and hydroxylamine hydrochloride $(150 \mathrm{mg}, 2.12 \mathrm{mmol})$ in pyridine (10 $\mathrm{ml})$ was refluxed until the reaction was complete (10-15 min, TLC control). The reaction mixture was cooled, diluted with chloroform $(100 \mathrm{ml})$; pyridine was removed by $5 \% \mathrm{HCl}$, and the acid was washed off with water until the neutral reaction of the washed water. The chloroform solution was dried over anhydrous $\mathrm{Na}_{2} \mathrm{SO}_{4}$ and evaporated, the residue was purified by chromatography on silica $\left(\mathrm{CCl}_{4}\right.$ :acetone $40: 1$ by volume). Oxim 3 was obtained as darkgreen crystals $\left(81 \mathrm{mg}\right.$, yield 55\%). $\mathrm{m} / \mathrm{z}(\mathrm{EI}): 563(100 \%)\left[(\mathrm{M})^{+}\right]$, $547(30 \%)$ [(MH-OH $\left.)^{+}\right], 476(38 \%) \quad\left[\left(\mathrm{M}^{-\mathrm{CH}_{2}} \mathrm{CH}_{2} \mathrm{CO}_{2} \mathrm{CH}_{3}\right)^{+}\right]$, $475(45 \%)\left[\left(\mathrm{MH}-\mathrm{H}_{2}-\mathrm{CH}_{2} \mathrm{CH}_{2} \mathrm{CO}_{2} \mathrm{CH}_{3}\right)^{+}\right], 459(35 \%)[(\mathrm{MH}-$ $\left.\left.\mathrm{CH}_{2} \mathrm{CH}_{2} \mathrm{CO}_{2} \mathrm{CH}_{3}-\mathrm{H}_{2} \mathrm{O}\right)^{+}\right]$. ${ }^{1} \mathrm{H}$ NMR $\left(\mathrm{CDCl}_{3}, 300 \mathrm{MHz}\right) \delta_{\mathrm{H}} \mathrm{ppm}$ : $9.79\left(1 \mathrm{H}, \mathrm{s}, \mathrm{H}^{10}\right), 8.98\left(1 \mathrm{H}, \mathrm{s}, \mathrm{H}^{5}\right), 8.90\left(1 \mathrm{H}, \mathrm{s}, \mathrm{H}^{20}\right), 8.15(1 \mathrm{H}, \mathrm{dd}$, $3-\left(\mathrm{CH}=\mathrm{CH}_{2}\right), J=18.0$ and $\left.11.6 \mathrm{~Hz}\right), 6.35\left(1 \mathrm{H}, \mathrm{d}, 3-\left(\mathrm{CH}=\mathrm{CH} H_{\text {trans }}\right)\right.$, $J=18.0 \mathrm{~Hz}), 6.21\left(1 \mathrm{H}, \mathrm{dd}, 3-\left(\mathrm{CH}=\mathrm{CH} H_{\mathrm{cis}}\right), J=11.6 \mathrm{~Hz}\right), 5.28(1 \mathrm{H}$, $\left.\mathrm{d}, \mathrm{H}^{13(2)}{ }_{\mathrm{A}}, J=20.0 \mathrm{~Hz}\right), 5.12\left(1 \mathrm{H}, \mathrm{d}, \mathrm{H}^{13(2)}{ }_{\mathrm{B}}, J=20.0 \mathrm{~Hz}\right), 4.67(1 \mathrm{H}$, q, $\left.\mathrm{H}^{18}, J=6.0 \mathrm{~Hz}\right), 4.55\left(1 \mathrm{H}\right.$, br d, $\left.\mathrm{H}^{17}, J=8.0 \mathrm{~Hz}\right), 3.64(2 \mathrm{H}, \mathrm{q}$, $\left.8-\left(\mathrm{CH}_{2} \mathrm{CH}_{3}\right), J=9.6 \mathrm{~Hz}\right), 3.63\left(3 \mathrm{H}, \mathrm{s}, 17-\left(\mathrm{CH}_{2} \mathrm{CH}_{2} \mathrm{COOCH}_{3}\right)\right), 3.52$ $\left(3 \mathrm{H}, \mathrm{s}, 12-\mathrm{CH}_{3}\right), 3.37\left(3 \mathrm{H}, \mathrm{s}, 2-\mathrm{CH}_{3}\right), 2.70\left(3 \mathrm{H}, \mathrm{s}, 7-\mathrm{CH}_{3}\right), 2.98-2.40$ $\left(4 \mathrm{H}, \mathrm{m}, 17-\left(\mathrm{CH}_{2} \mathrm{CH}_{2} \mathrm{COOCH}_{3}\right)\right), 1.89\left(3 \mathrm{H}, \mathrm{d}, 18-\mathrm{CH}_{3}, J=6.8 \mathrm{~Hz}\right)$, $1.56\left(3 \mathrm{H}, \mathrm{t}, 8-\mathrm{CH}_{2} \mathrm{CH}_{3}, J=7.2 \mathrm{~Hz}\right),-1.03(1 \mathrm{H}$, br s, I-NH), -2.68 $(1 \mathrm{H}$, br s, III-NH).

Methylpyropheophorbide a 13(1)-methoxim, 4. A solution of methylpyropheophorbide $a$ (2) $(100 \mathrm{mg}, 0.18 \mathrm{mmol})$ and methoxyamine hydrochloride $(300 \mathrm{mg}, 3.55 \mathrm{mmol})$ in pyridine $(6$ $\mathrm{ml})$ was refluxed until the reaction was complete (10-15 min, TLC control). The reaction mixture was treated analogously to procedure for oxim (3). The obtained mixture of chlorins was separated by chromatography on silica ( $\mathrm{CCl}_{4}$ :acetone $90: 1$ by volume). Methoxim 4 was obtained as dark green crystals (58 mg, $60 \%$ yield). $\mathrm{m} / \mathrm{z}(\mathrm{EI})$ : $577(99 \%)\left[(\mathrm{M})^{+}\right], 576(100 \%)\left[(\mathrm{M}-\mathrm{H})^{+}\right], 575(65 \%)\left[\left(\mathrm{M}-\mathrm{H}_{2}\right)^{+}\right]$, $546(27 \%)\left[\left(\mathrm{MH}-\mathrm{CH}_{3} \mathrm{OH}\right)^{+}\right], 490(10 \%)\left[\left(\mathrm{M}-\mathrm{CH}_{2} \mathrm{CH}_{2} \mathrm{CO}_{2} \mathrm{CH}_{3}\right)^{+}\right]$, $459(35 \%)$ [(MH- $\left.\left.\mathrm{CH}_{3} \mathrm{OH}-\mathrm{CH}_{2} \mathrm{CH}_{2} \mathrm{CO}_{2} \mathrm{CH}_{3}\right)^{+}\right], 458(30 \%)$ [(MH$\left.\left.\mathrm{CH}_{3} \mathrm{OH}-\mathrm{CH}_{2} \mathrm{CH}_{2} \mathrm{CO}_{2} \mathrm{CH}_{3}-\mathrm{H}\right)^{+}\right]$. ${ }^{1} \mathrm{H}$ NMR $\left(\mathrm{CDCl}_{3}, 300 \mathrm{MHz}\right) \delta_{\mathrm{H}}$ ppm: $9.69\left(1 \mathrm{H}, \mathrm{s}, \mathrm{H}^{10}\right), 9.57\left(1 \mathrm{H}, \mathrm{s}, \mathrm{H}^{5}\right), 8.79\left(1 \mathrm{H}, \mathrm{s}, \mathrm{H}^{20}\right), 8.12(1 \mathrm{H}, \mathrm{dd}$,
3- $\left(\mathrm{CH}=\mathrm{CH}_{2}\right), J=16.0$ and $\left.12.0 \mathrm{~Hz}\right), 6.30\left(1 \mathrm{H}, \mathrm{dd}, 3-\left(\mathrm{CH}=\mathrm{CH} H_{\text {trans }}\right)\right.$, $J=16.0$ and $0.5 \mathrm{~Hz}), 6.14\left(1 \mathrm{H}, \mathrm{dd}, 3-\left(\mathrm{CH}=\mathrm{CH}_{\text {cis }}\right), J=12.0\right.$ and $0.5 \mathrm{~Hz}), 5.55\left(1 \mathrm{H}, \mathrm{d}, \mathrm{H}^{13(2)}, J=20.0 \mathrm{~Hz}\right), 5.43^{\mathrm{cis}}\left(1 \mathrm{H}, \mathrm{d}, \mathrm{H}^{13(2)}\right.$ $J=20.0 \mathrm{~Hz}), 4.59\left(1 \mathrm{H}, \mathrm{q}, \stackrel{\mathrm{A}}{\mathrm{H}}^{18}, J=8.0 \mathrm{~Hz}\right), 4.40\left(1 \mathrm{H}, \mathrm{br} \mathrm{d}, \mathrm{H}^{\mathrm{B}}\right.$, $J=8.0 \mathrm{~Hz}), 4.31\left(3 \mathrm{H}, \mathrm{s}, 13(1)-\mathrm{NOCH}_{3}\right), 3.75\left(2 \mathrm{H}, \mathrm{q}, 8-\left(\mathrm{CH}_{2} \mathrm{CH}_{3}\right)\right.$, $J=8.0 \mathrm{~Hz}), 3.65\left(3 \mathrm{H}, \mathrm{s}, 17-\left(\mathrm{CH}_{2} \mathrm{CH}_{2} \mathrm{COOCH}_{3}\right)\right), 3.57(3 \mathrm{H}, \mathrm{s}, 12-$ $\left.\mathrm{CH}_{3}\right), 3.49\left(3 \mathrm{H}, \mathrm{s}, 2-\mathrm{CH}_{3}\right), 3.31\left(3 \mathrm{H}, \mathrm{s}, 7-\mathrm{CH}_{3}\right), 2.78-2.20(4 \mathrm{H}, \mathrm{m}$, $\left.17-\left(\mathrm{CH}_{2} \mathrm{CH}_{2} \mathrm{COOCH}_{3}\right)\right), 1.82\left(3 \mathrm{H}, \mathrm{d}, 18-\mathrm{CH}_{3}, J=8.0 \mathrm{~Hz}\right), 1.71(3 \mathrm{H}$, t, $\left.8-\mathrm{CH}_{2} \mathrm{CH}_{3}, J=8.0 \mathrm{~Hz}\right),-0.81(1 \mathrm{H}$, br s, I-NH), $-2.82(1 \mathrm{H}$, br s, III-NH).

Zinc methylpyropheophorbide a, Zn-2. A solution of zinc acetate $(210 \mathrm{mg}, 1.15 \mathrm{mmol})$ in methanol $(5 \mathrm{ml})$ was added to a solution of methylpyropheophorbide $a$ (2) $(75 \mathrm{mg}, 0.14 \mathrm{mmol})$ in chloroform $(5 \mathrm{ml})$. The mixture was stirred for $60 \mathrm{~min}$. The reaction mixture was diluted with chloroform $(50 \mathrm{ml})$, washed with water to remove the excess of zinc acetate and methanol, dried over anhydrous $\mathrm{Na}_{2} \mathrm{SO}_{4}$ and solvent was evaporated under reduced pressure. The residue after evaporation was purified by chromatography on silica $\left(\mathrm{CCl}_{4}\right.$ :acetone 50:1 - 1:1). The darkgreen crystals of $\mathbf{Z n - 2}$ (52 mg) were obtained with yield 77\%. UVvis $\left(\mathrm{CHCl}_{3}\right) \lambda \mathrm{nm}$ (relative intensity, \%): 658(46), 611(7), 571(1), 524(3), 428(100). ${ }^{1} \mathrm{H}$ NMR $\left(\mathrm{CDCl}_{3}, 300 \mathrm{MHz}\right) \delta_{\mathrm{H}} \mathrm{ppm}: 8.98(1 \mathrm{H}, \mathrm{s}$, $\left.\mathrm{H}^{10}\right), 8.89\left(1 \mathrm{H}, \mathrm{s}, \mathrm{H}^{5}\right), 8.36\left(1 \mathrm{H}, \mathrm{s}, \mathrm{H}^{20}\right), 7.86\left(1 \mathrm{H}, \mathrm{dd}, 3-\left(\mathrm{CH}=\mathrm{CH}_{2}\right)\right.$, $J=17.6$ and $11.2 \mathrm{~Hz}), 6.12\left(1 \mathrm{H}, \mathrm{dd}, 3-\left(\mathrm{CH}=\mathrm{CH}_{\text {trans }}\right), J=17.6\right.$ and $1.6 \mathrm{~Hz}), 6.03\left(1 \mathrm{H}, \mathrm{dd}, 3-\left(\mathrm{CH}=\mathrm{CH} H_{\mathrm{cis}}\right), J=11.6\right.$ and $\left.1.6 \mathrm{~Hz}\right), 4.72$ $\left(1 \mathrm{H}, \mathrm{d}, \mathrm{H}^{13(2)}{ }_{\mathrm{A}}, J=20.0 \mathrm{~Hz}\right), 4.63\left(1 \mathrm{H}, \mathrm{d}, \mathrm{H}^{13(2)}{ }_{\mathrm{B}}, J=20.0 \mathrm{~Hz}\right), 4.37$ $\left(1 \mathrm{H}, \mathrm{q}, \mathrm{H}^{18}, J=8.0 \mathrm{~Hz}\right), 3.98\left(1 \mathrm{H}\right.$, br d, $\left.\mathrm{H}^{17}, J=8.0 \mathrm{~Hz}\right), 3.35(2 \mathrm{H}$, $\left.\mathrm{m}, 8-\left(\mathrm{CH}_{2} \mathrm{CH}_{3}\right)\right), 3.42\left(3 \mathrm{H}, \mathrm{s}, 17-\left(\mathrm{CH}_{2} \mathrm{CH}_{2} \mathrm{COOCH}_{3}\right)\right), 3.36(3 \mathrm{H}, \mathrm{s}$, $\left.12-\mathrm{CH}_{3}\right), 3.30\left(3 \mathrm{H}, \mathrm{s}, 2-\mathrm{CH}_{3}\right), 2.94\left(3 \mathrm{H}, \mathrm{s}, 7-\mathrm{CH}_{3}\right), 2.44-2.15(4 \mathrm{H}$, $\left.\mathrm{m}, 17-\left(\mathrm{CH}_{2} \mathrm{CH}_{2} \mathrm{COOCH}_{3}\right)\right), 1.83\left(3 \mathrm{H}, \mathrm{d}, 18-\mathrm{CH}_{3}, J=6.8 \mathrm{~Hz}\right), 1.49$ $\left(3 \mathrm{H}, \mathrm{t}, 8-\mathrm{CH}_{2} \mathrm{CH}_{3}, J=7.6 \mathrm{~Hz}\right.$ ).

Zinc methylpyropheophorbide a 13(1)-oxim, Zn-3, was prepared as described above for $\mathbf{Z n - 2}$ complex from ligand 3 (75 $\mathrm{mg}, 0.13 \mathrm{mmol})$ and zinc acetate $(202 \mathrm{mg}, 1.10 \mathrm{mmol})$. The darkgreen crystals of $\mathbf{Z n - 3}$ were obtained ( $45 \mathrm{mg}, 56 \%$ yield). UVvis $\left(\mathrm{CHCl}_{3}\right) \lambda \mathrm{nm}$ (relative intensity, \%): 651(52), 595(6), 555(1), 508(4), 417(100). ${ }^{1} \mathrm{H} \mathrm{NMR}\left(\mathrm{CDCl}_{3}, 300 \mathrm{MHz}\right) \delta_{\mathrm{H}} \mathrm{ppm}: 9.53(1 \mathrm{H}, \mathrm{s}$, $\left.\mathrm{H}^{10}\right), 9.46\left(1 \mathrm{H}, \mathrm{s}, \mathrm{H}^{5}\right), 8.66\left(1 \mathrm{H}, \mathrm{s}, \mathrm{H}^{20}\right), 8.12\left(1 \mathrm{H}, \mathrm{dd}, 3-\left(\mathrm{CH}=\mathrm{CH}_{2}\right)\right.$, $J=18.0$ and $12.0 \mathrm{~Hz}), 6.24\left(1 \mathrm{H}, \mathrm{dd}, 3-\left(\mathrm{CH}=\mathrm{CH} H_{\text {trans }}\right), J=18.0\right.$ and $1.5 \mathrm{~Hz}), 6.10\left(1 \mathrm{H}, \mathrm{dd}, 3-\left(\mathrm{CH}=\mathrm{CH} H_{\text {cis }}\right), J=12.0\right.$ and $\left.1.0 \mathrm{~Hz}\right), 5.52$ $\left(1 \mathrm{H}, \mathrm{d}, \mathrm{H}^{13(2)}{ }_{\mathrm{A}}, J=18.0 \mathrm{~Hz}\right), 5.41\left(1 \mathrm{H}, \mathrm{d}, \mathrm{H}^{13(2)}{ }_{\mathrm{B}}, J=18.0 \mathrm{~Hz}\right), 4.40$ $\left(1 \mathrm{H}\right.$, br d, $\left.\mathrm{H}^{17}, J=7.0 \mathrm{~Hz}\right), 3.74\left(1 \mathrm{H}, \mathrm{q}, \mathrm{H}^{18}, J=9.0 \mathrm{~Hz}\right), 3.74(2 \mathrm{H}, \mathrm{q}$, $\left.8-\left(\mathrm{CH}_{2} \mathrm{CH}_{3}\right), J=9.0 \mathrm{~Hz}\right), 3.59\left(3 \mathrm{H}, \mathrm{s}, 17-\left(\mathrm{CH}_{2} \mathrm{CH}_{2} \mathrm{COOCH}_{3}\right)\right), 3.51$ $\left(3 \mathrm{H}, \mathrm{s}, 12-\mathrm{CH}_{3}\right), 3.46\left(3 \mathrm{H}, \mathrm{s}, 2-\mathrm{CH}_{3}\right), 3.29\left(3 \mathrm{H}, \mathrm{s}, 7-\mathrm{CH}_{3}\right), 2.84-2.17$ $\left(4 \mathrm{H}, \mathrm{m}, 17-\left(\mathrm{CH}_{2} \mathrm{CH}_{2} \mathrm{COOCH}_{3}\right)\right), 1.89\left(3 \mathrm{H}, \mathrm{d}, 18-\mathrm{CH}_{3}, J=6.0 \mathrm{~Hz}\right)$, $1.71\left(3 \mathrm{H}, \mathrm{t}, 8-\mathrm{CH}_{2} \mathrm{CH}_{3}, J=7.6 \mathrm{~Hz}\right)$.

Zinc methylpyropheophorbide a 13(1)-methoxim, Zn-4, was prepared as described above for $\mathbf{Z n - 2}$ complex from ligand $4(34 \mathrm{mg}, 0.06 \mathrm{mmol})$ and zinc acetate $(100 \mathrm{mg}, 0.55 \mathrm{mmol})$. The dark-green crystals of $\mathbf{Z n - 4}$ were obtained (18 mg, $47 \%$ yield). UV-vis $\left(\mathrm{CHCl}_{3}\right) \lambda \mathrm{nm}$ (relative intensity, \%): 652(54), 598(5), 558(1), 510(4), 419(100). ${ }^{1} \mathrm{H} \mathrm{NMR}\left(\mathrm{CDCl}_{3}, 300 \mathrm{MHz}\right) \delta_{\mathrm{H}} \mathrm{ppm}: 9.50(1 \mathrm{H}, \mathrm{s}$, $\left.\mathrm{H}^{10}\right), 9.46\left(1 \mathrm{H}, \mathrm{s}, \mathrm{H}^{5}\right), 8.65\left(1 \mathrm{H}, \mathrm{s}, \mathrm{H}^{20}\right), 8.11\left(1 \mathrm{H}, \mathrm{dd}, 3-\left(\mathrm{CH}=\mathrm{CH}_{2}\right)\right.$, $J=18.0$ and $12.0 \mathrm{~Hz}), 6.24\left(1 \mathrm{H}, \mathrm{dd}, 3-\left(\mathrm{CH}=\mathrm{CH} H_{\text {trans }}\right), J=18.0\right.$ and $3.0 \mathrm{~Hz}), 6.10\left(1 \mathrm{H}, \mathrm{dd}, 3-\left(\mathrm{CH}=\mathrm{CH} H_{\mathrm{cis}}\right), J=12.0\right.$ and $\left.3.0 \mathrm{~Hz}\right)$, $5.45\left(1 \mathrm{H}, \mathrm{d}, \mathrm{H}^{13(2)}{ }_{\mathrm{A}}, J=21.0 \mathrm{~Hz}\right), 5.34\left(1 \mathrm{H}, \mathrm{d}, \mathrm{H}^{13(2)}{ }_{\mathrm{B}}, J=21.0 \mathrm{~Hz}\right)$, $4.30\left(3 \mathrm{H}, \mathrm{s}, 13(1)-\mathrm{NOCH}_{3}\right), 4.63-4.53\left(1 \mathrm{H}, \mathrm{m}, \mathrm{H}^{17}, \mathrm{H}^{18}\right), 3.73(2 \mathrm{H}, \mathrm{q}$, 8- $\left.\left(\mathrm{CH}_{2} \mathrm{CH}_{3}\right), J=9.0 \mathrm{~Hz}\right), 3.63\left(3 \mathrm{H}, \mathrm{s}, 17-\left(\mathrm{CH}_{2} \mathrm{CH}_{2} \mathrm{COOCH}_{3}\right)\right), 3.56$ $\left(3 \mathrm{H}, \mathrm{s}, 12-\mathrm{CH}_{3}\right), 3.45\left(3 \mathrm{H}, \mathrm{s}, 2-\mathrm{CH}_{3}\right), 3.28\left(3 \mathrm{H}, \mathrm{s}, 7-\mathrm{CH}_{3}\right), 2.80-2.20$ $\left(4 \mathrm{H}, \mathrm{m}, 17-\left(\mathrm{CH}_{2} \mathrm{CH}_{2} \mathrm{COOCH}_{3}\right)\right), 1.89\left(3 \mathrm{H}, \mathrm{d}, 18-\mathrm{CH}_{3}, J=6.0 \mathrm{~Hz}\right)$, $1.71\left(3 \mathrm{H}, \mathrm{t}, 8-\mathrm{CH}_{2} \mathrm{CH}_{3}, J=6.0 \mathrm{~Hz}\right)$.

3(1), 3(2)-Bis (N, N-dimethylaminomethyl)methylpyropheophorbide a, 5. Bis( $N, N$-dimethylamino)methane (1) $(1.5 \mathrm{ml}, 11.70 \mathrm{mmol})$ was added to the solution of $\mathrm{Zn}-2$ complex (72 $\mathrm{mg}, 0.12 \mathrm{mmol})$ in the mixture of THF $(3 \mathrm{ml})$ and glacial acetic acid $(3 \mathrm{ml})$. The reaction mixture was kept for $90 \mathrm{~min}$ at the room temperature, then it was diluted with chloroform $(50 \mathrm{ml})$ and washed with water. After addition of conc. hydrochloric 
acid $(8 \mathrm{ml})$ the mixture was allowed to stay for $20 \mathrm{~min}$, then $50 \mathrm{ml}$ of chloroform was added to the mixture, it was washed with water until a neutral reaction, dried by anhydrous $\mathrm{Na}_{2} \mathrm{SO}_{4}$, and the solvent was thoroughly evaporated under reduced pressure at $30-40^{\circ} \mathrm{C}$. The residue was purified by chromatography on silica $\left(\mathrm{CCl}_{4}\right.$ :acetone 50:1 - 1:1, then chloroform:ethanol 30:1-1:1). The fractions, containing the goal product, were collected, evaporated and reprecipitated from the mixture of chloroform and pentane. The dark-green crystals of 5 were obtained ( $36 \mathrm{mg}, 40 \%$ yield). $m / z$ (EI): $665(5 \%)\left[\left(\mathrm{MH}+\mathrm{H}_{2}\right)^{+}\right], 664(30 \%)\left[\left(\mathrm{M}+\mathrm{H}_{2}\right)^{+}\right], 663(60 \%)$ $\left[(\mathrm{MH})^{+}\right], 648(100 \%)\left[\left(\mathrm{M}-\mathrm{CH}_{3}\right)^{+}\right], 607\left[\left(\mathrm{M}+\mathrm{H}_{2}-\mathrm{CH}_{2} \mathrm{~N}\left(\mathrm{CH}_{3}\right)_{2}\right)^{+}\right], 579$ $(80 \%)\left[\left(\mathrm{M}+\mathrm{H}_{2}-\mathrm{CH}_{2} \mathrm{CH}_{2} \mathrm{CO}_{2} \mathrm{CH}_{3}\right)^{+}\right]$. IR $(\mathrm{KBr}) v_{\max } \mathrm{cm}^{-1}: 1616.4 \mathrm{~m}$ («chlorin's bond»), $1695.4 \mathrm{~s}\left(v_{\mathrm{C}=0}, 13(1)-\mathrm{keto}\right), 1737.9 \mathrm{~s}\left(v_{\mathrm{C}=0}\right.$, ester), $2767.9 \mathrm{w}, 2818.0 \mathrm{w}\left(\mathrm{v}_{\mathrm{C}-\mathrm{H}}, \mathrm{CH}_{2} \mathrm{~N}\left(\mathrm{CH}_{3}\right)_{2}\right), 2866.2 \mathrm{~m}, 2927.9 \mathrm{~m}, 2960.7$ $\mathrm{m},\left(v_{\mathrm{C}-\mathrm{H}}\right.$, alkyl $\left.\mathrm{CH}_{2}, \mathrm{CH}_{3}\right){ }^{1} \mathrm{H}$ NMR $\left(\mathrm{CDCl}_{3}, 300 \mathrm{MHz}\right) \delta_{\mathrm{H}} \mathrm{ppm}: 9.68$ $\left(1 \mathrm{H}, \mathrm{s}, \mathrm{H}^{10}\right), 9.56\left(1 \mathrm{H}, \mathrm{s}, \mathrm{H}^{5}\right), 8.59\left(1 \mathrm{H}, \mathrm{s}, \mathrm{H}^{20}\right), 7.40-7.20(1 \mathrm{H}, \mathrm{m}, 3$ $\left.-\mathrm{C}\left(\mathrm{CH}_{2} \mathrm{~N}\left(\mathrm{CH}_{3}\right)_{2}\right)=\mathrm{CH}\left(\mathrm{CH}_{2} \mathrm{~N}\left(\mathrm{CH}_{3}\right)_{2}\right)\right), 5.30\left(1 \mathrm{H}, \mathrm{d}, \mathrm{H}^{13(2)}{ }_{\mathrm{A}}, J=18.0\right.$ $\mathrm{Hz}), 5.14\left(1 \mathrm{H}, \mathrm{d}, \mathrm{H}^{13(2)}{ }_{\mathrm{B}}, J=18.0 \mathrm{~Hz}\right), 4.52\left(1 \mathrm{H}, \mathrm{q}, \mathrm{H}^{18}, J=6.0 \mathrm{~Hz}\right)$, $4.33\left(1 \mathrm{H}\right.$, br d, $\left.\mathrm{H}^{17}, J=9.0 \mathrm{~Hz}\right), 3.80-3.67\left(2 \mathrm{H}, \mathrm{m}, 8-\left(\mathrm{CH}_{2} \mathrm{CH}_{3}\right)\right)$, 3.60-3.00 (4H, m, 3-C $\left.\left(\mathrm{CH}_{2} \mathrm{~N}\left(\mathrm{CH}_{3}\right)_{2}\right)=\mathrm{CH}\left(\mathrm{CH}_{2} \mathrm{~N}\left(\mathrm{CH}_{3}\right)_{2}\right)\right), 3.70(3 \mathrm{H}$, s, $\left.17-\left(\mathrm{CH}_{2} \mathrm{CH}_{2} \mathrm{COOCH}_{3}\right)\right), 3.63\left(3 \mathrm{H}, \mathrm{s}, 12-\mathrm{CH}_{3}\right), 3.48\left(3 \mathrm{H}, \mathrm{s}, 2-\mathrm{CH}_{3}\right)$, $3.31\left(3 \mathrm{H}, \mathrm{s}, 7-\mathrm{CH}_{3}\right), 2.71-2.41\left(4 \mathrm{H}, \mathrm{m}, 17-\left(\mathrm{CH}_{2} \mathrm{CH}_{2} \mathrm{COOCH}_{3}\right)\right), 1.84$ $\left(3 \mathrm{H}, \mathrm{t}, 8-\mathrm{CH}_{2} \mathrm{CH}_{3}, J=7.6 \mathrm{~Hz}\right), 1.75 / 1.72\left(3 \mathrm{H}, \mathrm{d}, 18-\mathrm{CH}_{3}, J=6.8\right.$ $\mathrm{Hz}), 0.34(1 \mathrm{H}$, br s, I-NH), -1.81 (1H, br s, III-NH).

3(1),3(2)-Bis (N,N-dimethylaminomethyl) methylpyropheophorbide a 13(1)-oxim, 6, was prepared as described above for 5. The action of bis( $N, N$-dimethylamino)methane (1) $(1.0 \mathrm{ml}, 7.80 \mathrm{mmol})$ on $\mathbf{Z n - 3}$ complex $(35 \mathrm{mg}, 0.06 \mathrm{mmol})$ in the mixture of THF $(2 \mathrm{ml})$ and glacial acetic acid $(2 \mathrm{ml})$ gave compound 6 (14 mg, $32 \%$ yield) as dark-green crystals. $m / z$ (EI): 680 (3\%) $\left[\left(\mathrm{MH}+\mathrm{H}_{2}\right)^{+}\right], 679(5 \%)\left[\left(\mathrm{M}+\mathrm{H}_{2}\right)^{+}\right], 663(80 \%)\left[\left(\mathrm{MH}+\mathrm{H}_{2}-\mathrm{OH}\right)^{+}\right]$, $648(100 \%)\left[\left(\mathrm{MH}+\mathrm{H}_{2}-\mathrm{OH}-\mathrm{CH}_{3}\right)^{+}\right]$. IR $(\mathrm{KBr}) v_{\max } \mathrm{cm}^{-1}: 1612.5 \mathrm{~m}$ («chlorin's bond»), $1639.5 \mathrm{~s}\left(v_{\mathrm{C}=\mathrm{N}}\right.$, oxim), $1732.1 \mathrm{~s}\left(v_{\mathrm{C}=\mathrm{O}}\right.$, ester), $2727.4 \mathrm{w}\left(v_{\mathrm{C}-\mathrm{H}}, \mathrm{CH}_{2} \mathrm{~N}\left(\mathrm{CH}_{3}\right)_{2}\right), 2868.1 \mathrm{~m}, 2927.9 \mathrm{~m}, 2960.7 \mathrm{~m} \mathrm{(v} \mathrm{v}_{\mathrm{C}-\mathrm{H}}$, alkyl $\left.\mathrm{CH}_{2}, \mathrm{CH}_{3}\right)$. ${ }^{2} \mathrm{H} \mathrm{NMR}\left(\mathrm{CDCl}_{3}, 300 \mathrm{MHz}\right) \delta_{\mathrm{H}} \mathrm{ppm}: 9.90(1 \mathrm{H}$, s, $\left.\mathrm{H}^{10}\right), 9.33 / 9.29\left(1 \mathrm{H}, \mathrm{s}, \mathrm{H}^{5}\right), 8.84\left(1 \mathrm{H}, \mathrm{s}, \mathrm{H}^{20}\right), 7.46-7.36(1 \mathrm{H}, \mathrm{m}, 3$ $\left.-\mathrm{C}\left(\mathrm{CH}_{2} \mathrm{~N}\left(\mathrm{CH}_{3}\right)_{2}\right)=\mathrm{C} H\left(\mathrm{CH}_{2} \mathrm{~N}\left(\mathrm{CH}_{3}\right)_{2}\right)\right), 5.48\left(1 \mathrm{H}, \mathrm{d}, \mathrm{H}^{13(2)}{ }_{\mathrm{A}}, J=18.0\right.$ $\mathrm{Hz}), 5.37\left(1 \mathrm{H}, \mathrm{d}, \mathrm{H}^{13(2)}{ }_{\mathrm{B}}, J=18.0 \mathrm{~Hz}\right), 4.55\left(1 \mathrm{H}, \mathrm{m}, \mathrm{H}^{18}\right), 4.33(1 \mathrm{H}$, m, $\left.\mathrm{H}^{17}\right), 3.79-3.68\left(2 \mathrm{H}, \mathrm{m}, 8-\left(\mathrm{CH}_{2} \mathrm{CH}_{3}\right)\right), 3.60-3.00(4 \mathrm{H}, \mathrm{m}, 3-\mathrm{C}(\mathrm{CH}$ $\left.\left.{ }_{2} \mathrm{~N}\left(\mathrm{CH}_{3}\right)_{2}\right)=\mathrm{CH}\left(\mathrm{CH}_{2} \mathrm{~N}\left(\mathrm{CH}_{3}\right)_{2}\right)\right), 3.63\left(3 \mathrm{H}, \mathrm{s}, 17-\left(\mathrm{CH}_{2} \mathrm{CH}_{2} \mathrm{COOCH}_{3}\right)\right)$, $3.62\left(3 \mathrm{H}, \mathrm{s}, 12-\mathrm{CH}_{3}\right), 3.61\left(3 \mathrm{H}, \mathrm{s}, 2-\mathrm{CH}_{3}\right), 3.44\left(3 \mathrm{H}, \mathrm{s}, 7-\mathrm{CH}_{3}\right), 2.31-$ $2.26\left(4 \mathrm{H}, \mathrm{m}, 17-\left(\mathrm{CH}_{2} \mathrm{CH}_{2} \mathrm{COOCH}_{3}\right)\right), 1.83\left(3 \mathrm{H}, \mathrm{d}, 18-\mathrm{CH}_{3}, J=6.8\right.$ $\mathrm{Hz}), 1.70\left(3 \mathrm{H}, \mathrm{t}, 8-\mathrm{CH}_{2} \mathrm{CH}_{3}, J=7.6 \mathrm{~Hz}\right),-1.30(1 \mathrm{H}$, br s, I-NH), -3.01/-3.04 (1H, br s, III-NH)

3(1),3(2)-Bis (N,N-dimethylaminomethyl)methylpyropheophorbide a 13(1)-methoxim, 7, was prepared as described above for 5. The action of bis( $N, N$-dimethylamino)methane (1) $(0.5 \mathrm{ml}, 3.90 \mathrm{mmol})$ on $\mathbf{Z n}-4$ complex $(10 \mathrm{mg}, 0.02 \mathrm{mmol})$ in the mixture of THF $(2 \mathrm{ml})$ and glacial acetic acid $(2 \mathrm{ml})$ gave compound 7 (5 mg, $30 \%$ yield) as dark-green crystals. $\mathrm{m} / \mathrm{z}$ (EI): $694(3 \%)\left[\left(\mathrm{MH}+\mathrm{H}_{2}\right)^{+}\right], 663(1 \%)\left[\left(\mathrm{M}+\mathrm{H}_{2}-\mathrm{OCH}_{3}\right)^{+}\right], 635(30 \%)$ $\left[\left(\mathrm{M}+\mathrm{H}_{2}-\mathrm{CH}_{2} \mathrm{~N}\left(\mathrm{CH}_{3}\right)_{2}\right)^{+}\right], 607(80 \%)\left[\left(\mathrm{MH}+\mathrm{H}_{2}-\mathrm{CH}_{2} \mathrm{CH}_{2} \mathrm{CO}_{2} \mathrm{CH}_{3}\right)^{+}\right]$, $579(100 \%)\left[\left(\mathrm{M}+\mathrm{H}_{2}-2 \mathrm{CH}_{2} \mathrm{~N}\left(\mathrm{CH}_{3}\right)_{2}\right)^{+}\right]$. IR $(\mathrm{KBr}) \mathrm{v}_{\max } \mathrm{cm}^{-1}: 1608.6$ $\mathrm{m}$ («chlorin's bond»), $1629.9 \mathrm{~s}\left(v_{\mathrm{C}=\mathrm{N}}\right.$, oxim), $1735.9 \mathrm{~s}\left(v_{\mathrm{C}=\mathrm{O}}\right.$, ester), $2816.6 \mathrm{w}\left(v_{\mathrm{C}-\mathrm{H}}, \mathrm{CH}_{2} \mathrm{~N}\left(\mathrm{CH}_{3}\right)_{2}\right), 2868.2 \mathrm{~m}, 2929.9 \mathrm{~m}, 2958.8 \mathrm{~m},\left(\mathrm{v}_{\mathrm{C}-\mathrm{H}}\right.$, alkyl $\left.\mathrm{CH}_{2}, \mathrm{CH}_{3}\right) .{ }^{2} \mathrm{H}$ NMR $\left(\mathrm{CDCl}_{3}, 300 \mathrm{MHz}\right) \delta_{\mathrm{H}} \mathrm{ppm}: 9.90 / 9.89$ $\left(1 \mathrm{H}, \mathrm{s}, \mathrm{H}^{10}\right), 9.72\left(1 \mathrm{H}, \mathrm{s}, \mathrm{H}^{5}\right), 8.89\left(1 \mathrm{H}, \mathrm{s}, \mathrm{H}^{20}\right), 7.44-7.37(1 \mathrm{H}, \mathrm{m}$, $\left.3-\mathrm{C}\left(\mathrm{CH}_{2} \mathrm{~N}\left(\mathrm{CH}_{3}\right)_{2}\right)=\mathrm{CH}\left(\mathrm{CH}_{2} \mathrm{~N}\left(\mathrm{CH}_{3}\right)_{2}\right)\right), 5.62\left(1 \mathrm{H}, \mathrm{d}, \mathrm{H}^{13(2)}{ }_{\mathrm{A}}, J=18.0\right.$ $\mathrm{Hz}), 5.50\left(1 \mathrm{H}, \mathrm{d}, \mathrm{H}^{13(2)}{ }_{\mathrm{B}}, J=18.0 \mathrm{~Hz}\right), 4.66\left(1 \mathrm{H}, \mathrm{q}, \mathrm{H}^{18}, J=6.0\right.$ $\mathrm{Hz}), 4.33\left(1 \mathrm{H}, \mathrm{br} \mathrm{d}, \mathrm{H}^{17}, J=9.0 \mathrm{HZ}\right), 4.36\left(3 \mathrm{H}, \mathrm{s}, 13(1)-\mathrm{NOCH}_{3}\right)$, $3.86\left(2 \mathrm{H}, \mathrm{q}, 8-\left(\mathrm{CH}_{2} \mathrm{CH}_{3}\right), J=8.0 \mathrm{~Hz}\right), 3.60-3.00\left(4 \mathrm{H}, \mathrm{m}, 3-\mathrm{C}\left(\mathrm{CH}_{2} \mathrm{~N}\right.\right.$ $\left.\left.\left(\mathrm{CH}_{3}\right)_{2}\right)=\mathrm{CH}\left(\mathrm{CH}_{2} \mathrm{~N}\left(\mathrm{CH}_{3}\right)_{2}\right)\right), 3.75\left(3 \mathrm{H}, \mathrm{s}, 17-\left(\mathrm{CH}_{2} \mathrm{CH}_{2} \mathrm{COOCH}_{3}\right)\right)$, $3.64\left(3 \mathrm{H}, \mathrm{s}, 12-\mathrm{CH}_{3}\right), 3.61\left(3 \mathrm{H}, \mathrm{s}, 2-\mathrm{CH}_{3}\right), 3.45\left(3 \mathrm{H}^{2}, \mathrm{~s}, 7-\mathrm{CH}_{3}\right), 2.71-$ $2.41\left(4 \mathrm{H}, \mathrm{m}, 17-\left(\mathrm{CH}_{2} \mathrm{CH}_{2} \mathrm{COOCH}_{3}\right)\right), 1.87\left(3 \mathrm{H}, \mathrm{d}, 18-\mathrm{CH}_{3}, J=8.0\right.$ $\mathrm{Hz}), 1.70\left(3 \mathrm{H}, \mathrm{t}, 8-\mathrm{CH}_{2} \mathrm{CH}_{3}, J=7.0 \mathrm{~Hz}\right),-1.27(1 \mathrm{H}$, br s, I-NH), $-2.92(1 \mathrm{H}, \mathrm{III}-\mathrm{NH})$.

Interaction of methylpyropheophorbide a (2) with bisamine (1) at room temperature. $\operatorname{Bis}(N, N$-dimethylamino) methane (1) $(1.5 \mathrm{ml}, 11.70 \mathrm{mmol})$ was added to the solution of methylpyropheophorbide $a(2)(56 \mathrm{mg}, 0.10 \mathrm{mmol})$ in the mixture of THF $(4 \mathrm{ml})$ and glacial acetic acid $(4 \mathrm{ml})$. This reaction mixture was allowed to stay for 5 days at the room temperature, then it was diluted with chloroform $(50 \mathrm{ml})$ and washed with water. The isolation of a product from the reaction mixture was carried out similarly to that of compound $\mathbf{5}$. The initial compound $\mathbf{2}(42 \mathrm{mg})$ and the reaction product $\mathbf{8}(6 \mathrm{mg})$ were isolated as the dark-green crystals. $m / z$ (ESI): $618\left[\left(\mathrm{MH}-\mathrm{HN}\left(\mathrm{CH}_{3}\right)_{2}\right)^{+}\right], 561\left[\left(\mathrm{MH}-\mathrm{HN}\left(\mathrm{CH}_{3}\right)_{2}-\right.\right.$ $\left.\left.\mathrm{CH}_{2} \mathrm{~N}\left(\mathrm{CH}_{3}\right)_{2}\right)^{+}\right]$. IR (KBr) $v_{\text {max }} \mathrm{cm}^{-1}: 1611 \mathrm{~m}$ («chlorin's bond»), $1711 \mathrm{c}\left(v_{\mathrm{C}=0}\right.$, ketone), $1737.9 \mathrm{~s}\left(v_{\mathrm{C}=0}\right.$, ester), $2765.9 \mathrm{w}$ and $2818.0 \mathrm{w}$ $\left(v_{\mathrm{C}-\mathrm{H}}, \mathrm{CH}_{2}\left(\mathrm{NCH}_{3}\right)_{2}\right), 2864.3,2927.9$ and $2956.9\left(v_{\mathrm{C}-\mathrm{H}}\right.$, alkyl $\mathrm{CH}_{2}$, $\left.\mathrm{CH}_{3}\right) .{ }^{1} \mathrm{H}$ NMR $\left(\mathrm{CDCl}_{3}, 300 \mathrm{MHz}\right) \delta_{\mathrm{H}} \mathrm{ppm}: 9.65\left(1 \mathrm{H}, \mathrm{s}, \mathrm{H}^{10}\right), 9.49$ $\left(1 \mathrm{H}, \mathrm{s}, \mathrm{H}^{5}\right), 8.67\left(1 \mathrm{H}, \mathrm{s}, \mathrm{H}^{20}\right), 8.04\left(1 \mathrm{H}, \mathrm{dd}, 3-\left(\mathrm{CH}=\mathrm{CH}_{2}\right), J=18.0\right.$ and $11.5 \mathrm{~Hz}), 6.32\left(1 \mathrm{H}, \mathrm{d}, 3-\left(\mathrm{CH}=\mathrm{CH} H_{\text {trans }}\right), J=18.5 \mathrm{~Hz}\right), 6.20$ $\left(1 \mathrm{H}, \mathrm{d}, 3-\left(\mathrm{CH}=\mathrm{CH} H_{\mathrm{cis}}\right), J=11.5 \mathrm{~Hz}\right), 4.63-4.50\left(2 \mathrm{H}, \mathrm{m}, \mathrm{H}^{18}, \mathrm{H}^{17}\right)$, $3.78\left(3 \mathrm{H}, \mathrm{s}, 17-\left(\mathrm{CH}_{2} \mathrm{CH}_{2} \mathrm{COOCH}_{3}\right)\right), 3.58\left(3 \mathrm{H}, \mathrm{s}, 12-\mathrm{CH}_{3}\right), 3.46$ $\left(3 \mathrm{H}, \mathrm{s}, 2-\mathrm{CH}_{3}\right), 3.26\left(3 \mathrm{H}, \mathrm{s}, 7-\mathrm{CH}_{3}\right), 3.75-3.00\left(6 \mathrm{H}, \mathrm{m}, 8-\left(\mathrm{CH}_{2} \mathrm{CH}_{3}\right)\right.$, $\left.3-\mathrm{C}\left(\mathrm{CH}_{2} \mathrm{~N}\left(\mathrm{CH}_{3}\right)_{2}\right)=\mathrm{CH}\left(\mathrm{CH}_{2} \mathrm{~N}\left(\mathrm{CH}_{3}\right)_{2}\right)\right), \quad 2.62-2.46 \quad(2 \mathrm{H}, \quad \mathrm{m}$, 17- $\left.\left(\mathrm{CH}_{2} \mathrm{CH}_{2} \mathrm{COOCH}_{3}\right)\right), 2.42-2.25\left(2 \mathrm{H}, \mathrm{m}, 17-\left(\mathrm{CH}_{2} \mathrm{CH}_{2} \mathrm{COOCH}_{3}\right)\right)$, 1.91-2.25 $\left(12 \mathrm{H}, \mathrm{m}, 3-\mathrm{C}\left(\mathrm{CH}_{2} \mathrm{~N}\left(\mathrm{CH}_{3}\right)_{2}\right)=\mathrm{CH}\left(\mathrm{CH}_{2} \mathrm{~N}\left(\mathrm{CH}_{3}\right)_{2}\right)\right), 1.75-1.67$ $\left(6 \mathrm{H}, \mathrm{m}, 18-\mathrm{CH}_{3}, 8-\mathrm{CH}_{2} \mathrm{CH}_{3},\right),-0.15(1 \mathrm{H}$, br s, I-NH), $-1.87(1 \mathrm{H}$, br s, III-NH).

\section{Results and Discussion}

The interaction of phorbine derivatives of chlorophyll (2-4, Scheme 1) with bis( $N, N$-dimethylamino)methane (1) in the conditions of aminomethylation of chlorin $\mathrm{e}_{6} 13$-amides (refluxing in THF-AcOH) leads to formation of a complex mixture of several chlorins, the main component of which is the aminomethylation product. However, hence the yield of chlorin mixture in all cases does not exceed $20 \%$, the preparative obtaining of aminomethylated phorbine derivatives by a such method is extremely difficult. After chromatography the aminomethylation products (5-7) were isolated from the mixtures with 3-5\% yields. Probably, such a result is due to decreased reactivity of the vinyl group, caused by the presence of electron acceptor in an exocycle, as well as by side reactions with participation of the exocycle. The study of interaction of bis( $N, N$-dimethylamino)methane with methylpheophorbide $a$ shows, ${ }^{[6,7]}$ that dimethylaminomethyl cation can be generated from bisamine (1) in the more mild conditions (THF-AcOH, $10-12^{\circ} \mathrm{C}$ ). However, the study of the interaction of phorbine derivatives (2-4) with 1 in THF$\mathrm{AcOH}$ mixture at room temperature indicates that the lacking of the vinyl group's activity is in its aminomethylation under these conditions. Upon action of bisamine (1) on methylpheophorbide $a$ (2) aminomethylation of exocycle takes place (Scheme 1, derivative 8), the conversion of initial $\mathbf{1}$ and yield aminomethylated derivative is not high. The structure of aminomethylation product was established from the data of IR and ${ }^{1} \mathrm{H}$ NMR spectroscopy, as well as massspectroscopy. The presence of dimethylamine substituents in the molecule is evidenced by the IR and ${ }^{1} \mathrm{H}$ NMR spectra (see Experimental part). There are no any signals of protons in the 13(2) position of exocycle in ${ }^{1} \mathrm{H}$ NMR spectrum of the product, what indicates double substitution. The presence of bulky substituents in the exocycle is indirectly confirmed by the considerable ( $\mathrm{ca} 10 \mathrm{~cm}^{-1}$ ) displacement of the 13(1)keto group stretching vibrations in the IR spectrum of $\mathbf{8}$ if compared with that of initial compound. The low stability of the studied compound does not allow to observe the 
formation of molecular ions (ionization by elecron impact and in the electrospray conditions), but the fragmentation peaks of ions corresponding to the splitting of dimethylamine molecule and dimethylaminomethyl fragment from $\mathrm{MH}^{+}$are present. The formation of the exocycle aminomethylation product can be explained by the possibility of enol formation, which readily reacts with dimethylaminomethyl cation (Scheme 1). Changing of keto group by oxim or methoxim groups, from the one side, can increase the reactivity of the vinyl group, and, from the another side, prevent keto-enol tautomerisation, promoting condensation. That's why, for aminomethylation of the vinyl group, oxim $\mathbf{3}$ and methoxim 4 of methylpyropheophorbide $a$ are more favorable, than the initial keton $\mathbf{2}$. However oxim $\mathbf{3}$ and methoxim $\mathbf{4}$ do not react with bisamine $\mathbf{1}$ at room temperature. It is known that the insertion of zinc cation into the coordination sphere of chlorin increases the reactivity of vinyl group in the reactions with electrophiles and can allow the milder reaction conditions. This paper shows that aminomethylation of vinyl group in the zinc complexes of phorbine derivatives $\mathbf{2 - 4}$ can be performed at room temperature under action of bisamine $\mathbf{1}$ in the mixture of THF and AcOH. The corresponding metalfree double aminomethylated phorbine derivatives 5-7 can be obtained with $30-40 \%$ yields (in respect to the initial phorbines). In spite of several stages, the aminomethylation of the zinc complexes of phorbine surpasses a one-stage aminomethylation of metal-free derivatives in THF-AcOH under reflux not only by the higher yields of the final products, but also by the ease of treatment and separation of the obtained reaction mixtures. In spite of relative lability of zinc complexes, they are not demetallated in the reaction conditions. This fact allows to use an activating influence of zinc cation on the vinyl group and to prevent the site reactions. Metal-free aminomethylated derivatives 5-7 were obtained by demetallation of the aminomethylated products, formed from the zinc complexes, by addition of conc. hydrochloric acid directly in the reaction mixture without any isolation of aminomethylated complexes. We have not succeeded in the isolation of aminomethylated zinc complexes (Zn-5)-(Zn-7) because of their instability during the process of chromatography on silica they are fully decomposed with the formation of highly polar substances. The structure of the obtained metal-free aminomethylated derivatives 5-7 was confirmed by the IR, ${ }^{1} \mathrm{H}$ NMR spectroscopy and mass spectrometry data.
Aminomethylation of vinyl group in the zinc complexes of phorbine derivatives 2-4 proceeds analogously to that in 13-amides of chlorin $e_{6} \cdot{ }^{[3]}$ The differences in the IR and ${ }^{1} \mathrm{H}$ NMR spectra of aminomethylation products of phorbine derivatives and initial phorbines are similar to that described earlier for 13 -amides of chlorin $e_{6}{ }^{\left[{ }^{[3]}\right.}$ The presence of dimethylaminomethyl fragments is seen in the IR spectra (the $\mathrm{C}-\mathrm{H}$ stretching vibrations of $\mathrm{CH}_{2} \mathrm{~N}\left(\mathrm{CH}_{3}\right)_{2}$ fragment) and ${ }^{1} \mathrm{H}$ NMR spectra (singlets, corresponding to the methyl protons, and multiplets, corresponding to the methylene protons of $N, N$-dimethylaminomethyl substituents). The signals of two of three vinyl group protons are absent in the ${ }^{1} \mathrm{H}$ NMR spectra of aminomethylation products, thus evidencing about double substitution in the vinyl group. The study of aminomethylation products by ${ }^{1} \mathrm{H}$ NMR spectroscopy shows that, as in the case of aminomethylation of chlorin $e_{6} 13$-amides, the reaction results in the mixture of cis- and trans- isomers in 1:1 ratio, what is possible only at vicinal arrangement of $N, N$-dimethylaminomethyl substituents. This fact is also confirmed by the values of spin-spin interaction constants of the only vinyl group proton with methylene protons of dimethylaminomethyl substituent $\left(\mathrm{CH}_{2} \mathrm{NCH}_{3}\right)$. Splitting of triplets, corresponding to these protons in the both isomers, is $7.2-7.6 \mathrm{~Hz}$, what is possible only for 1,2-substituted vinyl group. It is interesting that according to the literature data ${ }^{[1,2]}$ aminomethylation of zinc complexes by dimethylmethyleneammonium iodide (Eschenmoser's reagent) (dry $\mathrm{CH}_{2} \mathrm{Cl}_{2}$, room temperature, 48 hrs) results in monoaminomethylated derivatives. In the case of 13 -amides of chlorin $e_{6}$ such a difference may be explained both by more drastic reaction conditions (refluxing of the reaction mixture) and by the fact, that mono-substitution under the action of bis( $N, N$-dimethylamino)methane (1) leads to the electroneutral substituent, which does not prevent a repeat attack of electrophile (Scheme 2).

In the case of Eschenmoser's reagent the cationic substituent is formed, preventing the interaction with the second dimethylaminomethyl cation (Scheme 2). The study of aminomethylation of phorbine zinc complexes 2-4 makes evident the determining role of the charge of the substituent inserted upon monosubstitution. At aminomethylation by bis( $N, N$-dimethylamino)methane $\mathbf{1}$ the repeat substitution proceeds so readily, that neither in the case of 13-amides of chlorin $e_{6}$ nor in the case of zinc complexes the products of monoaminomethylation of vinyl group were not found.

$$
\mathrm{CH}_{2}\left(\mathrm{NCH}_{3}\right)_{2}, \mathrm{THF} / \mathrm{AcOH}
$$

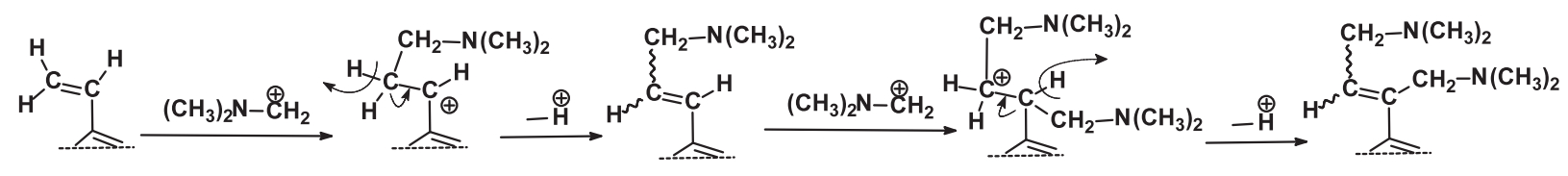

Eschenmoser's reagent using

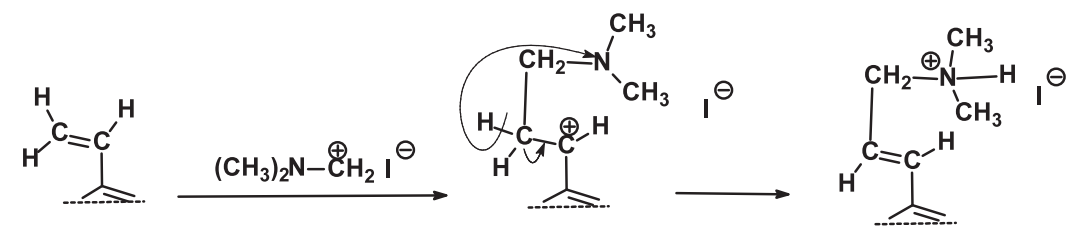

Scheme 2. 
Thriple aminomethylated derivatives were also not detected. Probably, their formation is prevented by the considerable steric problems, induced by two bulky dimethylaminomethyl groups.

\section{Conclusions}

We present herein a study indicating that the insertion of zinc cation into the chlorin's coordination sphere may be used for increase of the vinyl group activity in the reaction of aminomethylation with $\operatorname{bis}(N, N$-dimethylamino) methane (1) in the mixture of THF-AcOH. In spite of relative lability of of zinc complexes in acid media, their demetallation doesn't occur under conditions used for aminomethylation but easily proceeds after addition of hydrochloric acid. We have succeeded in extending the range of substrates of aminomethylation and obtained the new double aminomethylated derivatives. Related approach may be used for aminomethylation of other porphyrin compounds.

Acknowledgements. The work was supported by Federal Agency on Science and Innovations (state contract
№ 02.740.11.0081); by Russian Academy of Sciences (fundamental investigations, carried out together with organizations of the Siberian and Far-Eastern Branches of Russian Academy of Sciences, the state academies of sciences of Russia, national scientific academies of Community of Independent States and branch academies).

\section{References}

1. Ponomarev G.V. Chemistry of Heterocyclic Compounds 1997, 33, № 10, 1127-1166.

2. Pandey R.K., Shiau F.U., Smith N.N., Dougherty D.J., Smith K.M. Tetrahedron 1992, 48, 7591-7600.

3. Belykh D.V., Tarabukina I.S., Gruzdev I.V., Kodess M.I., Kutchin A.V. J. Porphyrins Phthalocyanines 2009, 13, 949956.

4. Belykh D.V., Tarabukina I.S., Matveev Yu.S., Kuchin A.V. Russ. J. Gen. Chem. 2007, 77, 1300-1307.

5. Ma L., Dolphin D. Tetrahedron 1996, 52, 849-860.

6. Belykh D.V., Tarabukina I.S., Gruzdev I.V., Kuchin A.V. Mend. Commun. 2007, 6, 340-342.

7. Belykh D.V., Tarabukina I.S., Gruzdev I.V., Kuchin A.V. Russ. J. Org. Chem. 2009, 45, 452-459.

Received 16.03.2010

Accepted 19.05.2010

First published on the web 02.07.2010 